Research Paper

\title{
A Canine Model of Femoral Head Osteonecrosis In- duced by an Ethanol Injection Navigated by a Novel Template
} Zhigang $\mathrm{Wu}^{1}$, Yong $\mathrm{Li}^{1}$, Zheng $\mathrm{Guo}^{1}{ }^{\boxplus}$

1. Department of Orthopedics, Xijing Hospital, Fourth Military Medical University, Xi'an, China;

2. Department of Orthopedics, General Hospital of Chengdu Military Region, Chengdu, China;

3. School of Stomatology, Fourth Military Medical University, Xi'an, China.

* These authors have contributed equally to this paper and are co-first authors.

$\triangle$ Corresponding author: Zheng Guo, MD, PhD. Address: Department of Orthopedics, Xijing Hospital, Fourth Military Medical University, No. 127, Changle West Road, Xi'an, Shaanxi Province, 710032, P.R. China. Tel: 86-29-84773411, Fax: 86-29-84773411 E-mail: guozheng@fmmu.edu.cn.

(C) Ivyspring International Publisher. This is an open-access article distributed under the terms of the Creative Commons License (http://creativecommons.org/ licenses/by-nc-nd/3.0/). Reproduction is permitted for personal, noncommercial use, provided that the article is in whole, unmodified, and properly cited.

Received: 2013.03.24; Accepted: 2013.08.13; Published: 2013.08.27

\begin{abstract}
There is no consensus on how to establish models of osteonecrosis of the femoral head (ONFH) in large mammals. The aim of this study was to investigate the effectiveness of a novel canine model of ONFH, induced by a navigated injection of absolute ethanol. Using three-dimensional reconstruction and rapid prototyping manufacturing techniques, a new template was designed and processed to navigate the ethanol injection. The femoral heads of 18 adult dogs were injected with ethanol. Macroscopic, X-ray and histological examinations were performed at 3, 6, and 9 weeks after the operation. Further, computed tomography (CT), magnetic resonance imaging (MRI), and radionuclide scans were performed 6 weeks postoperatively. Three weeks after the operation, the femoral heads showed evidence of osteonecrosis including increasing numbers of empty lacunae, decreased hematopoietic cells, and destroyed adipose tissue in the medullary cavity, which increased in severity at the subsequent follow-up evaluations at 6 and 9 weeks. Fractured trabeculae and fibrous tissue were noted 9 weeks postoperatively. Image analysis also revealed evidence of osteonecrosis, such as several osteopenic areas with sclerotic rims on the X-ray, several areas of low bone mineral density with sclerosis on the CT scan, increased uptake of the nuclide species in $\mathrm{MRI}$, and an inhomogeneous long T2 signal on the radioisotopic images. Ethanol injection navigated by our novel template was successful in establishing a canine model of ONFH. This model can be used to test new treatment modalities for human ONFH.
\end{abstract}

Key words: Animal model; Osteonecrosis of the femoral head; Ethanol; Injection; Canine.

\section{INTRODUCTION}

Osteonecrosis of the femoral head (ONFH) is a debilitating disease, which typically affects adults between the ages of 20 and 50 years [1]. It is estimated that between 10,000 and 20,000 new cases occur each year in the United States, accounting for $5 \%$ to $12 \%$ of patients that undergo total tip arthroplasty (THA) [2].
When treated conservatively, most affected hips (greater than $80 \%$ ) progress to femoral collapse and arthritis within 4 years of initial diagnosis, often necessitating THA [3]. However, the young age of patients with ONFH means that THA is unlikely to last the patient's lifetime. Thus, effective treatment mo- 
dalities are being actively sought that prevent collapse of affected femoral heads or prolong the interval between initial diagnosis and THA [1]. Current treatments for ONFH may be either pharmacotherapeutic or surgical, with no consensus on which treatment method is optimal. Surgical interventions include core decompression, vascularized or non-vascularized bone grafting, and osteotomy [1].

To develop new treatment modalities for ONFH, a variety of small animal models have been described, usually involving rats or rabbits. The majority of models induce ONFH by systematic insult, including steroid administration [4-7] or steroid combined with another adjunct agent [8-10]. However, to improve the relevance of ONFH animal models to the human condition and enable novel treatments to be evaluated, large mammal ONFH models are necessary.

Monggold et al. introduced a sheep model of ONFH induced by injection of ethanol [11]. Whilst this approach has been adopted and replicated [12-14], there are no reports on this application in canines. Both sheep and canines are usually used animals, which are especially suitable for testing procedures. Contrasting with sheep, canines are easily kept and somewhat not predisposed to fall sick in hot summer. Therefore, the aim of this study was to investigate the effectiveness of a novel canine model of ONFH induced by injection of absolute ethanol. In our preliminary experiment, we found that a single pass of the guide-wire is essential during the process of absolute ethanol injection, otherwise the ethanol leaks back out of the other tracks. Thus, an animal-specific template was adopted in this study to navigate the guide-wire instead of using $\mathrm{X}$-ray guidance.

\section{METHODS AND MATERIALS}

\section{Animals and experimental design}

This study was approved by the Institutional Animal Care and Use Committee of the Fourth Military Medical University. Eighteen adult dogs with a mean weight of $13.9 \mathrm{~kg}$ (range, 12.2-15.1 kg) and a mean age of 21.6 months (range, 17.7-24.1 months) were used. Each animal was raised in separate kennel of $3.24 \mathrm{~m}^{2}$, which allowed it to move freely.

The animals were randomly divided into three groups of six to be sacrificed at 3, 6, and 9 weeks, respectively. One of the femoral heads of each dog was randomly selected as 'experimental', in which the ethanol injection procedures were performed, and the contralateral femoral head was designated as the 'control', in which physiological saline was injected.

Postoperatively, the general condition of each animal was observed and documented, including eating and drinking habits, lameness, and mental state. X-ray examination was performed on each animal at 3, 6, and 9 weeks postoperatively, depending on the animal's group assignment. Animals in the second group (sacrificed at 6 weeks postoperatively) additionally underwent computed tomography (CT), radionuclide scanning, and magnetic resonance imaging (MRI). Following these investigations, each group of animals was sacrificed and the femoral heads were harvested. The femoral heads were then subjected to macroscopic and histological examination. In histological examination, the empty lacunae were counted and the extent of fibrosis was evaluated. Differences between groups were then statistically compared.

\section{The novel drilling template}

To navigate the drilling of the K-wire used to create the needle track for injecting the ethanol, a template was designed and manufactured. Firstly, a virtual three-dimensional (3D) reconstruction of the femoral head was produced (Figure 1A) using MIMICS 10.01 (Materialise's Interactive Medical Image Control System). Next, a shell of the 3D model was created and a virtual channel was placed in the optimal position and direction using Geomagic Studio 12[15] (Figure 1B, 1C and 1D). The template was then printed using a rapid prototyping manufacturing technique. In vitro and in vivo trials showed excellent agreement between the virtual and actual femoral heads (Figure 2A and 2B). Radiography confirmed that the K-wire navigated by the template was in the correct optimal position and the tip of the wire was at the center of the femoral head (Figure 2C).
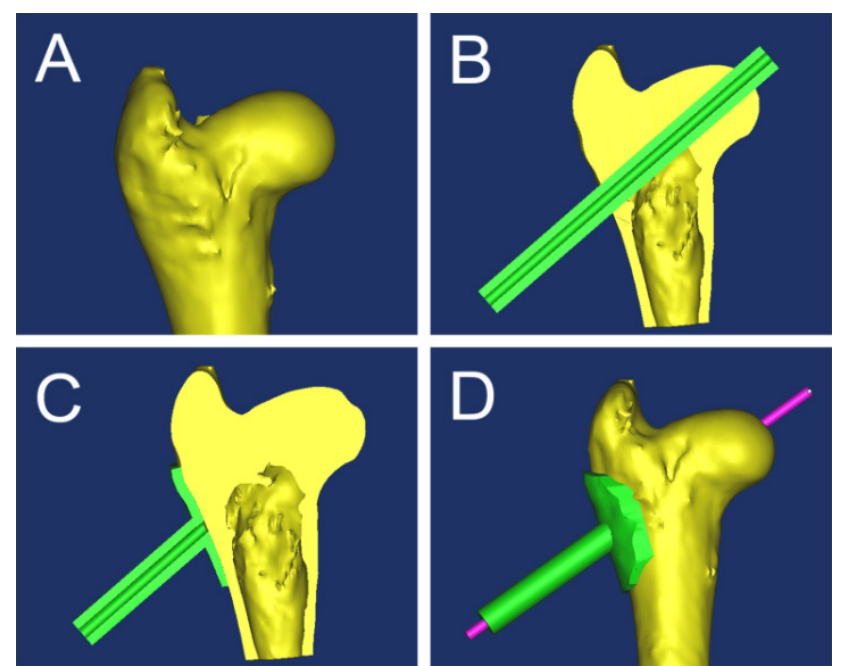

Figure I. A: three-dimensional (3D) reconstruction of the femoral head; B: a cannula was placed along the trajectory of guide-wire; C: the cannula was united with a shell created using Geomagic Studio $I 2$ and the cannula coinciding with the femoral head was removed; D: anteroposterior view of the jig and femoral head. 


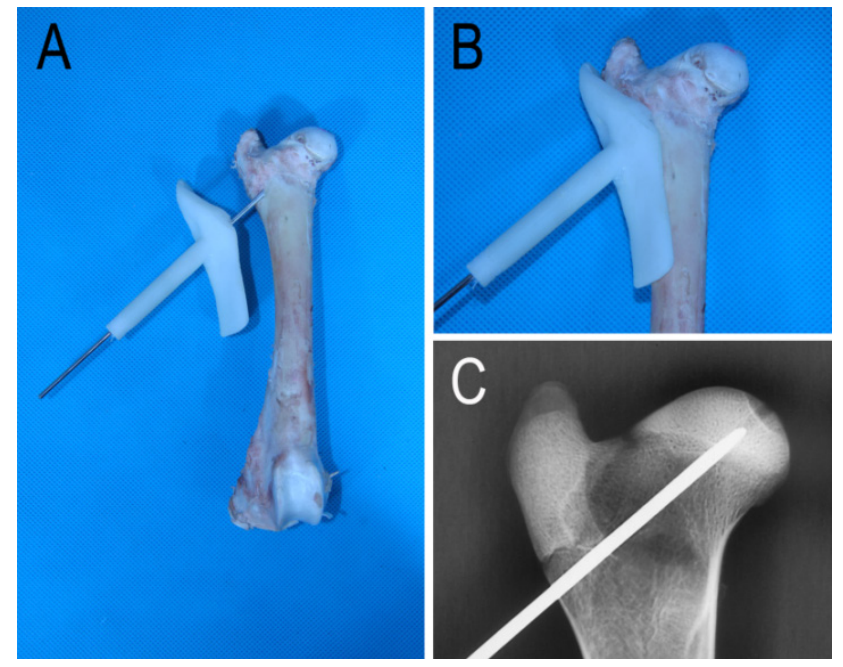

Figure 2. Drilling of the K-wire. A: the template was designed to guide the drilling of the K-wire; $\mathrm{B}$ : the template was well matched with the femur; $\mathrm{C}$ : $\mathrm{X}$-ray image of the $\mathrm{K}$-wire and the femoral head.

\section{Operative procedures}

After 12 hours fasting, xylazine hydrochloride (2 $\mathrm{mg} / \mathrm{kg}$ ) was administered intramuscularly for general anesthesia, which was maintained by intramuscular injections of sodium pentobarbital $(5 \mathrm{mg} / \mathrm{kg} \cdot \mathrm{h})$. The experimental hip of the animal was approached through a posterolateral incision under aseptic conditions. The biceps femoris muscle was reflected caudally, and the gluteal muscle group was detached from the major trochanter.

Using the navigating template, a $1.5 \mathrm{~mm}$ diameter K-wire was drilled into the center of the femoral head from the basal zone of the major trochanter. The length of K-wire drilled into the femur was congruent with pre-measured length in $3 \mathrm{D}$ reconstruction, ensuring that the distance between the tip of the K-wire and the articular surface was approximately $5 \mathrm{~mm}$ (Figure 3A). The K-wire was withdrawn and replaced with a $1.6 \mathrm{~mm}$ diameter cannulated needle. Four milliliters of absolute ethanol (> 99\%) was slowly injected into the femoral head with a pressure injector (Figure 3B, produced by Shanghai Kinetic Medical Corporation Limited) over a period of 20 minutes (0.2 $\mathrm{ml} / \mathrm{min}$ ).

The control femoral head was injected with physiological saline following the same procedures.

The surrounding soft tissue was protected with sponge pads. After the injection, the cortical hole induced by the K-wire was closed with bone wax, and the soft tissue around the trochanter region was irrigated with saline solution. The wound was closed in layers. The animal was then returned to the kennel and was allowed to roam freely in the kennel during the postoperative period.
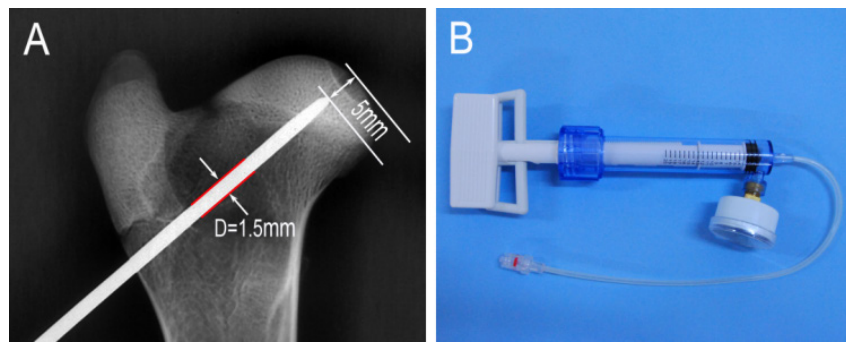

Figure $3 \mathrm{~A}$ : an illustration showing drilling of the K-wire; B: the pressure injector.

\section{The histological examination}

For histological examination, each animal was administered with excessive xylazine hydrochloride $(6 \mathrm{mg} / \mathrm{kg})$ intravenously and the femoral heads were harvested, then the femoral heads were cut into 3 mm-thick slices parallel to the femoral shaft. All specimens were fixed in 10\% formalin solution for 72 hours, and then decalcified in EDTA solution for 2 to 3 weeks. The slices were divided into four parts, designated I, II, III and IV (Figure 4A), and then each part was embedded into paraffin and cut into $5 \mu \mathrm{m}$ thick slices. The slices were stained with hematoxylin-eosin (H-E) and examined under a light microscope. The amounts of hematopoietic cells and fractured trabeculae, neogenic vessels as well as the grade of fibrosis were evaluated and documented by an experienced pathologist who was blinded to the experiment (level rank grade: none -, mild + , moderate ++ , much +++ ).

\section{Statistic analysis}

The high power images $(200 \times)$ of an area of 3 $\mathrm{mm} \times 3 \mathrm{~mm}$ (Figure 4B) in the center of each part were spliced and transformed into gray scale images. The empty lacunae were counted and the percentage of empty lacunae was calculated. The differences in the percentage of empty lacunae between each group were analyzed statistically using SPSS 16.0. The difference was considered to be significant when $\mathrm{P}<$ 0.05 .
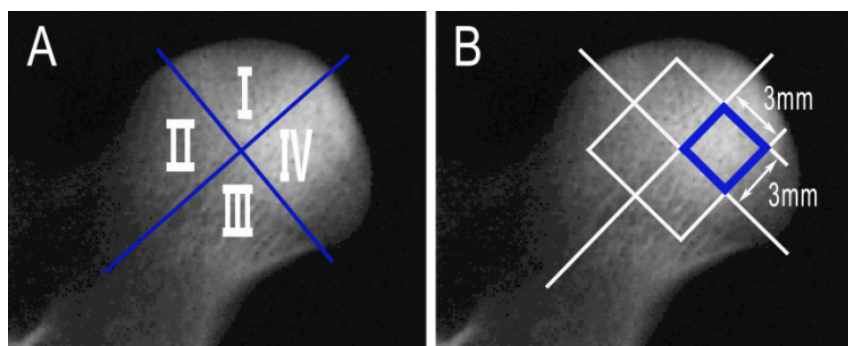

Figure $4 \mathrm{~A}$ : the slices were divided into four parts; B: the areas used to count empty lacunae. 


\section{RESULTS}

\section{General condition}

On postoperative days 1 and 2, the animals took a decreased amount of food and water, and the dogs preferred to lie rather than to stand. These conditions improved over time. The animals displayed signs of being crippled, with avoidance of weight bearing on the operated hips. One animal in the second group (sacrificed 6 weeks postoperatively) suffered a femoral neck fracture.

\section{X-ray examination}

Three weeks after the operation, six animals underwent X-ray examination. All of the operated femoral heads showed a different extent of inhomogeneous changes, and four of them showed several osteopenic areas (Figure 5B, blue arrows), which were accompanied with sclerotic rims (Figure 5B, white arrows). But, all of these changes were not obvious. At 6 weeks, two of the six operated femoral heads demonstrated mild segmental flattening (Figure 6B, black arrows). One animal developed a femoral head fracture (Figure 6C) on the experimental side. At 9 weeks, two of the operated femoral heads showed joint space narrowing with secondary osteoarthritis.

\section{$C T$, radionuclide and $M R I$ imaging}

Six weeks after the operation, five animals in group 2 underwent $\mathrm{CT}$ and radionuclide imaging. In $\mathrm{CT}$, there were several areas of low bone mineral density below the articular surface in the experimental femoral heads (Figure 7A, white arrow), which were accompanied with substantial sclerosis. Radioisotope imaging revealed an increased uptake of the nuclide species in the experimental femoral heads (figure 7B, white arrow). MRI examination showed an inhomogeneous long T2 signal in the experimental femoral heads, indicative of osteonecrosis (figure 7C and 7D, white arrow).

\section{Macroscopic examination}

The contour of the experimental femoral heads remained intact postoperatively, and osteonecrosis was indicated by heterogeneous color shades. Three weeks after the operation, the needle track still remained (Figure 8B, white arrow), and the color shade of the operated femoral heads became heterogeneous. Some regions of the section became lighter, but the majority of the section remained dark. At week 6, the lighter color predominated and the needle track was invisible. However, at 9 weeks postoperatively, the color of the specimen became darker than at 6 weeks.
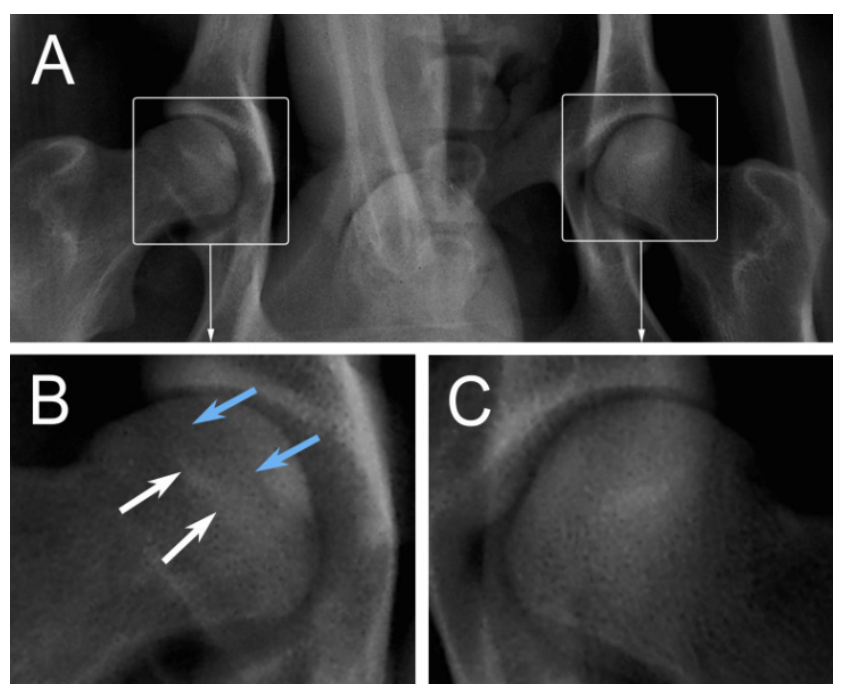

Figure 5. General changes seen in radiographic images. A: the anteroposterior X-ray examination of the hip; B: the experimental femoral head (blue arrows: osteopenic areas; white arrows: sclerotic rims); C: the control femoral head.

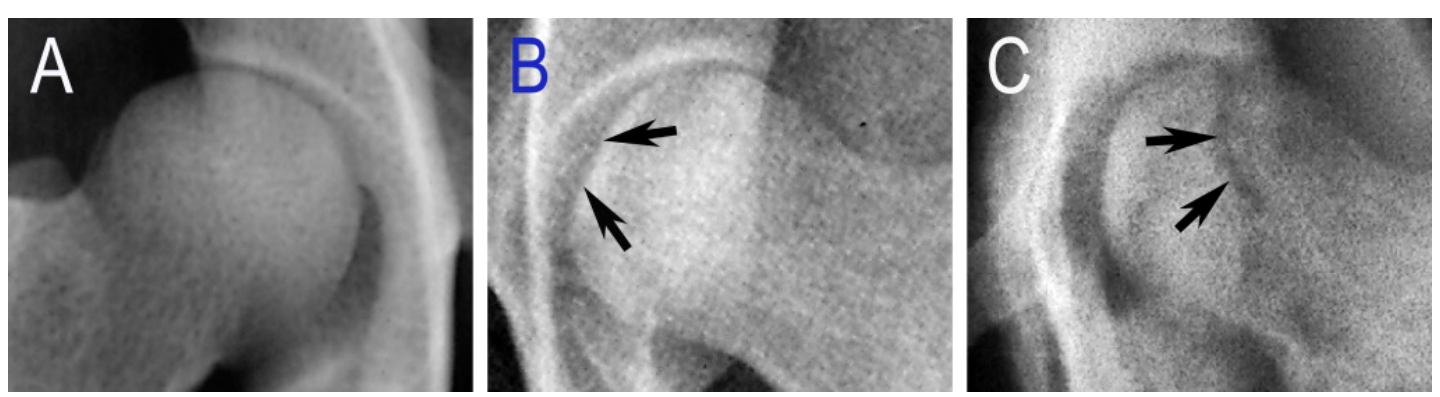

Figure 6. Specific changes seen in radiographic images. A: the control femoral head of control lateral; B: mild flattening of the articular cartilage (black arrows); C: a fractured femoral head (black arrows). 

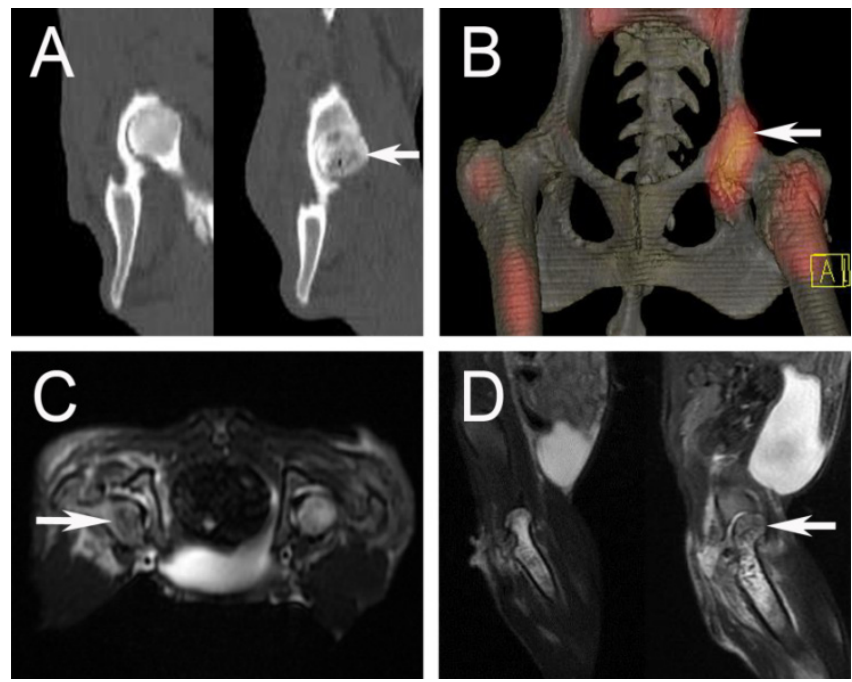

Figure 7. A: CT scan showed osteonecrosis in the right femoral head (white arrow); B: a "warm" change in radioisotope image (white arrow); C and D: inhomogeneous long T2 change in MRI examination (white arrow).

\section{Histological analysis}

Histological analysis of the control femoral heads showed that the trabeculae were intact and the the lacunae were filled with osteocytes (Figure 9A, black arrow). Hematopoietic cells (Figure 9A, red arrow) and adipose tissue (Figure 9A, green arrow) were abundant in the medullary canal. The structure of adiopose tissue was often intact. Three weeks after the operation, most of the lacunae in the experimental femoral heads showed little or no trabeculae in the necrotic area (Figure 9B, black arrow). Hematopoietic cells and adipose tissue in the medullary canal decreased, and the structure of adipose tissue broke down (Figure 9B, green arrow). At week 6, in some parts of the necrotic areas, fibroblast cells began to proliferate (Figure 9C, blue arrow) and the trabeculae began to resorb with occasional fractures (Figure 9C, green arrow). At week 6, the aforementioned changes were not dominant and empty lacunae were common. By 9 weeks, the marrow cavity was filled with

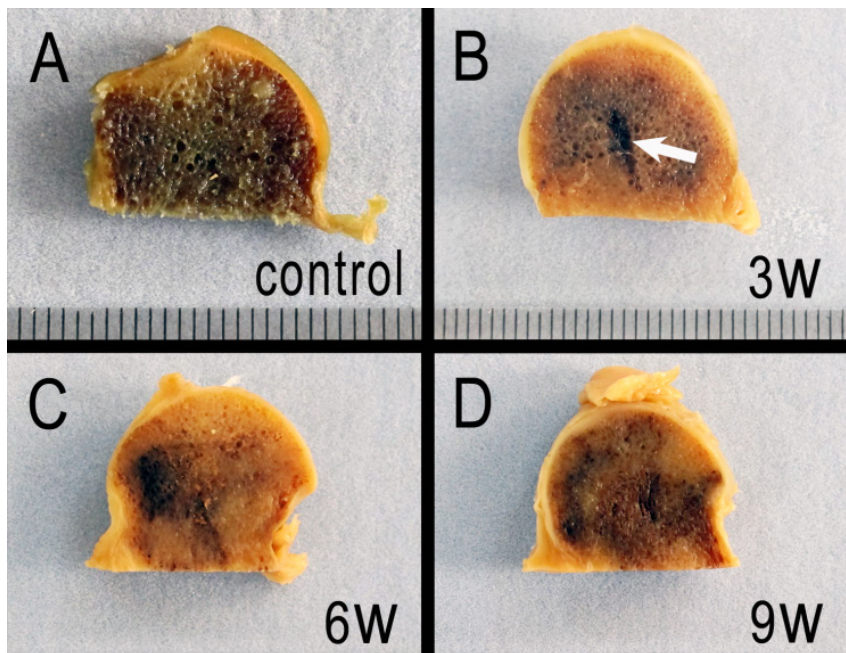

|1||||||||||||||||||||||1|||||||||||||||||||||||||||||||||||||||||||||1||||||||||||||||| Figure 8. Macroscopic examination. A: the control femoral head section. $B, C$ and $D$ : sections of necrotic femoral heads at week 3, 6, and 9, respectively.

proliferated fibroblast cells and the osteoclast cells were distributed along the margin of the trabeculae (Figure 9D, black arrow). Neogenic vessels filled with erythrocytes began to appear (Figure 9D, blue arrow). The amount of hematopoietic cells, neogenic vessels, and fractured trabeculae were evaluated and are documented in Table 1.

\section{Statistical analysis}

The percentage of empty lacunae in each group is shown as median (range) in Table 1. Nonparametric ranking tests showed that the percentage of empty lacunae in each operated group was higher than the corresponding control groups. The percentage of empty lacunae increased after the operation. However, 6 weeks after the operation, the percentage of empty lacunae did not increase. Statistical significance between two groups was set at $2 \times 0.05 / 4 \times$ $(4-1)=0.0083$.

Table I. The percentage of empty lacunae and histological changes (level rank grade: none -, mild+, moderate ++ , much +++ ) of the four groups.

\begin{tabular}{|c|c|c|c|c|c|c|}
\hline Time & $\begin{array}{l}\text { Median (range) of } \\
\text { empty lacunae per- } \\
\text { centage }\end{array}$ & $\begin{array}{l}\text { Difference between } \\
\text { control group and ex- } \\
\text { perimental groups }(p)\end{array}$ & $\begin{array}{l}\text { Hematopoie-tic } \\
\text { cells }\end{array}$ & $\begin{array}{l}\text { Fractured tra- } \\
\text { beculae }\end{array}$ & $\begin{array}{l}\text { Grade of fi- } \\
\text { brosis }\end{array}$ & $\begin{array}{l}\text { Neogenic } \\
\text { vessels }\end{array}$ \\
\hline Week 0 & $6.43(2.43)$ & & +++ & - & - & - \\
\hline Week 3 & $41.94(20.11)$ & 0.002 & + & - & - & - \\
\hline Week 6 & $61.23(8.96)$ & 0.004 & - & + & ++ & - \\
\hline Week 9 & $62.12(7.08)$ & 0.004 & - & ++ & +++ & + \\
\hline
\end{tabular}



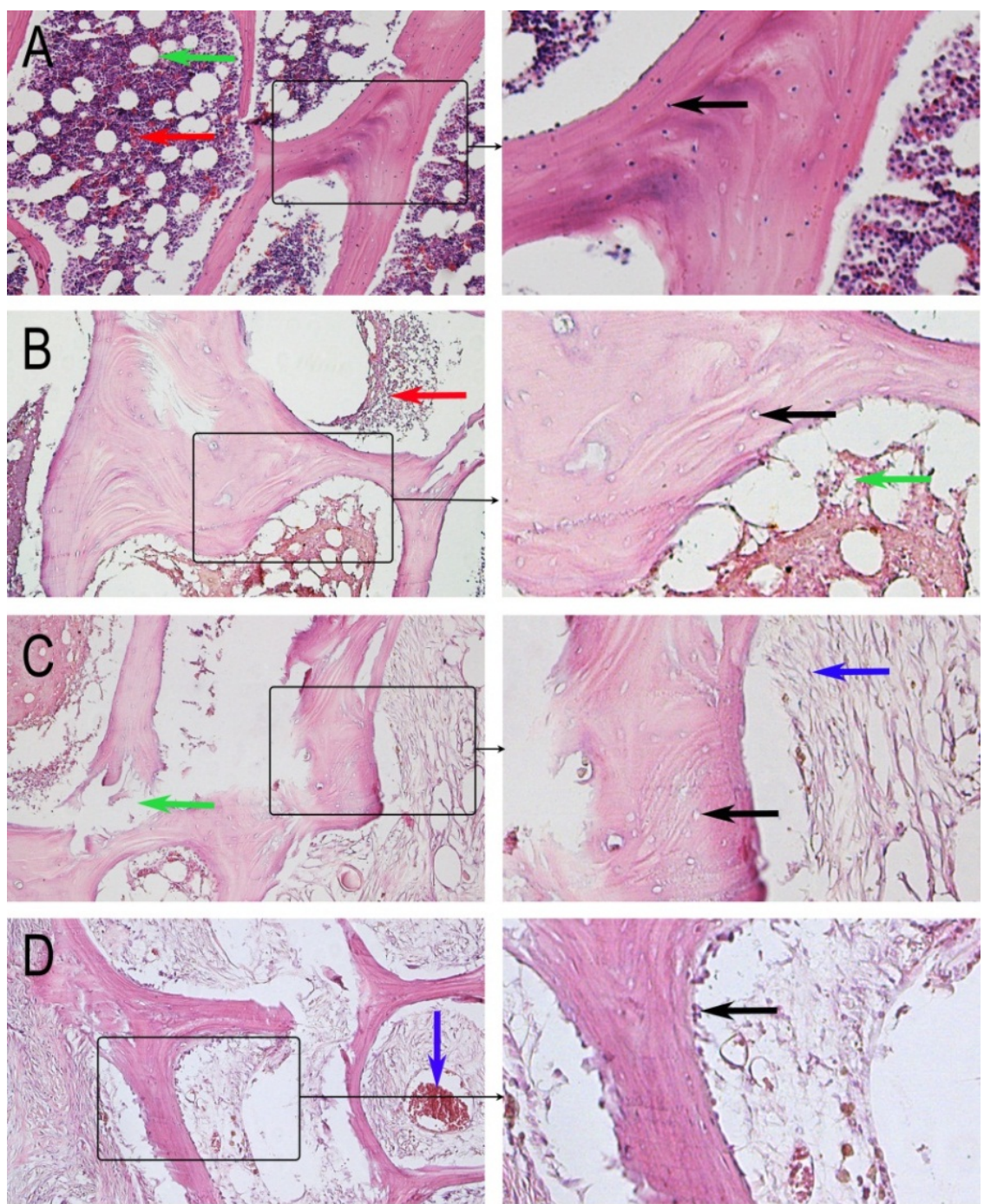

$50 \mu \mathrm{m}$

$20 \mu \mathrm{m}$

Figure 9. Histological microscopic pictures. A: control femoral heads (hematoxylin and eosin stain; black arrow: osteocyte in lacunae, green arrow: adipose tissue, red arrow: hematopoietic cell). B: experimental femoral heads at week 3 (black arrow: empty lacunae, green arrow: adipose tissue, red arrow: hematopoietic cell). C: 6 weeks after the operation (black arrow: empty lacunae, green arrow: fractured trabeculae, red arrow: hematopoietic cell, blue arrow: fibroblast cell). D: 6 weeks after the operation (black arrow: osteoclast cell, blue arrow: neogenic vessel).

\section{DISCUSSION}

This study investigated the effectiveness of establishing a canine model of ONFH induced by ethanol injection navigated by a novel template. The X-ray examination showed layers of low bone density below the cartilage of the femoral head, accompanied with layers of high bone density. These changes are similar to those that are seen with human ONFH. Hip axial CT and radionuclide examination showed obvious evidence of osteonecrosis in the experimental femoral heads, including substantial inhomogeneous sclerosis and increased uptake of the nuclide species. Further, histological examination revealed empty lacunae, fractured trabeculae, and abundant fibrocytes in the femoral heads injected with absolute ethanol. In the experimental femoral heads, the mean percentage of empty lacunae was higher than the $34 \%$ described by Gold EW [16], suggesting that our model was successful in inducing ONFH in the canine.

To produce a uniform necrotic area with anatomic proximity to the articular surface, as described by Malizos et al. [17], the tip of needle used for ethanol injection must be placed accurately in the upper medial femoral head (Figure 3A). Thus, we designed a template to navigate the drilling of the K-wire using a rapid prototyping manufacturing technique. X-ray examination confirmed that the tip of the K-wire was in ideal position (Figure 2C). Leakage of ethanol must be minimized during injection because leakage leads to a loss of accuracy in the prescribed dose and may also damage the surrounding soft tissue. Thus, we 
ensured that there was minimal clearance between the $\mathrm{K}$-wire (1.5 $\mathrm{mm}$ in diameter) and the cannulated needle $(1.6 \mathrm{~mm}$ in diameter). We also confirmed that there was minimal leakage with this protocol. There were difficulties using a standard injector, possibly due to the resistance caused by higher bone mineral density. We thus used a pressure injector (Figure 3B), which was effective.

Several large mammal models of ONFH have been proposed. These methods include cryogenic insult by liquid nitrogen [17-20], ethanol injection [11-14], osteotomy [21-24]. Among these methods, cryogenic insult and ethanol injection have been most commonly used; however, there is no consensus on the best model that will mimic human ONFH. Liquid nitrogen cryogenic insult has been a traditional method of inducing femoral head osteonecrosis, whereby a trapdoor is created to contain liquid nitrogen or a cryogenic insult probe. The diameter of this trapdoor ranged from $3 \mathrm{~mm}$ to $4.5 \mathrm{~mm}$ in previous studies $[19,25]$, which causes a large bone defect. This bone defect is non-negligible in animals with small diameter (12 to $18 \mathrm{~mm}$ ) femoral heads, such as dogs or rabbits. Further, this large bone defect is different from the pathology of human ONFH. Therefore, we suggest that it is not appropriate to use the cryogenic insult model for canines or rabbits.

Manggold et al. [11] introduced an ethanol injection animal model in Merino sheep for ONFH, which mimicked the early stage pathological changes of human ONFH. Zhu et al. [12] reproduced this model in Small Tail Han sheep, and some studies have adopted this method to test new treatment modalities of ONFH. However, until now, there have not been reports that test the suitability of this model in the canine. With the use of template navigating and pressure injecting, we were able to develop a sophisticated canine model of ONFH.

During the 9 week period of observation, femoral head collapse was not observed, possibly because the mechanical environment of the canine hip is different from that of the human. To mimic full stage pathological changes of human ONFH, Conzemius et al. [25-30] adopted the emu as an experimental animal for models of ONFH. However, the emu is a large, ostrich-like flightless bird that is hazardous to handle when awake. The emu is not a conventional experimental animal and is only found in Australia, decreasing its suitability for routine use as an animal for modeling ONFH.

A limitation of this study was the relatively small sample size, meaning that the follow-up observation period was limited. Additionally, one of the animals developed a femoral neck fracture 6 weeks after the operation (Figure 6C), which may have been caused by osteonecrosis of the femoral neck induced by inadequate ethanol infiltration. Further advances in the operative technique may avoid this complication.

In conclusion, an ethanol injection navigated using a novel template was successful in establishing a canine model of ONFH. This model can be used to test new treatment modalities of human ONFH.

\section{Acknowledgement}

This work was supported by the Natural Science Foundation of China (NSFC: 81171773 and 51271199) and Coordinative and Innovative Engineering Projects of Science and Technology of Shaanxi Province (No: 2012KTCG01-14).

\section{Competing Interests}

The authors have declared that no competing interest exists.

\section{References}

1 Marker DR, Seyler TM, Mcgrath MS, et al. Treatment of early stage osteonecrosis of the femoral head. J Bone Joint Surg Am. 2008; 90 Suppl 4: 175-187.

2 Mont MA, Hungerford DS. Non-traumatic avascular necrosis of the femoral head. J Bone Joint Surg Am. 1995; 77(3): 459-474.

3 Mont MA, Carbone JJ, Fairbank AC. Core decompression versus nonoperative management for osteonecrosis of the hip. Clin Orthop Relat Res. 1996;(324): 169-178.

4 Yang L, Boyd K, Kaste SC, et al. A mouse model for glucocorticoid-induced osteonecrosis: effect of a steroid holiday. J Orthop Res. 2009; 27(2): 169-175.

5 Sugano N, Kubo T, Takaoka K, et al. Diagnostic criteria for non-traumatic osteonecrosis of the femoral head. A multicentre study. J Bone Joint Surg Br. 1999; 81(4): 590-595.

6 Iwakiri K, Oda Y, Kaneshiro Y, et al. Effect of simvastatin on steroid-induced osteonecrosis evidenced by the serum lipid level and hepatic cytochrome P4503A in a rabbit model. J Orthop Sci. 2008; 13(5): 463-468.

7 Ichiseki T, Matsumoto T, Nishino M, et al. Oxidative stress and vascular permeability in steroid-induced osteonecrosis model. J Orthop Sci. 2004; 9(5): 509-515.

8 Yamamoto $\mathrm{T}$, Hirano $\mathrm{K}$, Tsutsui $\mathrm{H}$, et al. Corticosteroid enhances the experimental induction of osteonecrosis in rabbits with Shwartzman reaction. Clin Orthop Relat Res. 1995;(316): 235-243.

9 Wu X, Yang S, Duan D, et al. Experimental osteonecrosis induced by a combination of low-dose lipopolysaccharide and high-dose methylprednisolone in rabbits. Joint Bone Spine. 2008; 75(5): 573-578.

10 Qin L, Zhang G, Sheng H, et al. Multiple bioimaging modalities in evaluation of an experimental osteonecrosis induced by a combination of lipopolysaccharide and methylprednisolone. Bone. 2006; 39(4): 863-871.

11 Manggold J, Sergi C, Becker K, et al. A new animal model of femoral head necrosis induced by intraosseous injection of ethanol. Lab Anim. 2002; 36(2): 173-180.

$12 \mathrm{Zhu}$ ZH, Gao YS, Luo SH, et al. An animal model of femoral head osteonecrosis induced by a single injection of absolute alcohol: an experimental study. Med Sci Monit. 2011; 17(4): R97-R102.

13 Feitosa ML, Fadel L, Beltrao-Braga PC, et al. Successful transplant of mesenchymal stem cells in induced osteonecrosis of the ovine femoral head: preliminary results. Acta Cir Bras. 2010; 25(5): 416-422.

14 Yilmaz C, Golpinar A, Yilmaz N, et al. Comparison of bony healing and cartilage degeneration following strut grafting and trapdoor procedures in an ovine model. Orthopedics. 2008; 31(7): 656.

$15 \mathrm{Lu} \mathrm{S}, \mathrm{Xu} \mathrm{YQ}, \mathrm{Lu} W W$, et al. A novel patient-specific navigational template for cervical pedicle screw placement. Spine (Phila Pa 1976) 2009; 34 : E959-E966.

16 Gold EW, Fox OD, Weissfeld S, et al. Corticosteroid-induced avascular necrosis: an experimental study in rabbits. Clin Orthop Relat Res. 1978;(135): 272-280 
17 Malizos KN, Quarles LD, Seaber AV, et al. An experimental canine model of osteonecrosis: characterization of the repair process. J Orthop Res. 1993; 11(3): 350-357.

18 Nadel SN, Debatin JF, Richardson WJ, et al. Detection of acute avascular necrosis of the femoral head in dogs: dynamic contrast-enhanced MR imaging vs spin-echo and STIR sequences. AJR Am J Roentgenol. 1992; 159(6): 1255-1261.

19 Bowers JR, Dailiana ZH, Mccarthy EF, et al. Drug therapy increases bone density in osteonecrosis of the femoral head in canines. J Surg Orthop Adv. 2004; 13(4): 210-216.

20 Takaoka K, Yoshioka T, Hosoya T, et al. The repair process in experimentally induced avascular necrosis of the femoral head in dogs. Arch Orthop Trauma Surg. 1981; 99(2): 109-115.

21 Bonfiglio M. Aseptic necrosis of the femoral head in dogs; effect of drilling and bone grafting. Surg Gynecol Obstet. 1954; 98(5): 591-599.

22 Yan Z, Hang D, Guo C, et al. Fate of mesenchymal stem cells transplanted to osteonecrosis of femoral head. J Orthop Res. 2009; 27(4): 442-446.

23 Seiler JR, Kregor PJ, Conrad ER, et al. Posttraumatic osteonecrosis in a swine model. Correlation of blood cell flux, MRI and histology. Acta Orthop Scand. 1996; 67(3): 249-254.

24 Swiontkowski MF, Tepic S, Rahn BA, et al. The effect of fracture on femoral head blood flow. Osteonecrosis and revascularization studied in miniature swine. Acta Orthop Scand. 1993; 64(2): 196-202.

25 Conzemius MG, Brown TD, Zhang Y, et al. A new animal model of femoral head osteonecrosis: one that progresses to human-like mechanical failure. J Orthop Res. 2002; 20(2): 303-309.

26 Troy KL, Brown TD, Conzemius MG. Contact stress distributions on the femoral head of the emu (Dromaius novaehollandiae). J Biomech. 2009; 42(15): 2495-2500.

27 Goetz JE, Pedersen DR, Robinson DA, et al. The apparent critical isotherm for cryoinsult-induced osteonecrotic lesions in emu femoral heads. J Biomech. 2008; 41(10): 2197-2205.

28 Troy KL, Lundberg HJ, Conzemius MG, et al. Habitual hip joint activity level of the penned EMU (Dromaius novaehollandie). Iowa Orthop J. 2007; 27: 17-23.

29 Reed KL, Conzemius MG, Robinson RA, et al. Osteocyte-based image analysis for quantitation of histologically apparent femoral head osteonecrosis: application to an emu model. Comput Methods Biomech Biomed Engin. 2004; 7(1): 25-32.

30 Reed KL, Brown TD, Conzemius MG. Focal cryogen insults for inducing segmental osteonecrosis: computational and experimental assessments of thermal fields. J Biomech. 2003; 36(9): 1317-1326. 